\title{
Individual-Level Heterogeneity in Mask Wearing during the COVID-19 Pandemic in Malaysia
}

\author{
Stephen X. Zhang, ${ }^{1 *} \dagger$ Kim Hoe Looi, ${ }^{2} \dagger$ Nicolas Li, ${ }^{3}$ Xue Wan, ${ }^{4}$ and Jizhen Li ${ }^{5}$ \\ ${ }^{1}$ University of Adelaide, Adelaide, South Australia, Australia; ${ }^{2}$ Xiamen University Malaysia, Sunsuria City, Sepang, Selangor, Malaysia; ${ }^{3}$ University of \\ Dundee, Dundee, Scotland, United Kingdom; ${ }^{4}$ Tongji University, Shanghai, China; ${ }^{5}$ Tsinghua University, Beijing, China
}

\begin{abstract}
Wearing a face mask has been a key approach to contain or slow down the spread of COVID-19 in the ongoing pandemic. However, there is huge heterogeneity among individuals in their willingness to wear face masks during an epidemic. This research aims to investigate the individual heterogeneity to wear face masks and its associated predictors during the COVID-19 pandemic when mask-wearing was not mandatory. Based on a survey of 708 Malaysian adults and a multivariate least-squares fitting analysis, the results reveal a significant variance among individuals in wearing masks, as $34 \%$ of the individual adults did not always wear masks in public places. Female individuals, individuals who wash their hands more frequently, and those who reported more availability of personal protective equipment were more likely to practice maskwearing. The identification of less-compliant groups of mask wearing has critical implications by enabling more specific health communication campaigns.
\end{abstract}

Since the WHO declared COVID-19 a pandemic, the primary nonpharmaceutical containments at the individual level includes wearing face masks, improved hygiene, and other physical barriers. Health agencies worldwide recommended wearing face masks in public during the COVID-19 pandemic. For example, the CDC recommended: "Cover your mouth and nose with a mask when around others." 1 The WHO recommended medical/surgical face masks as a key measure of infection prevention and control against the transmission of COVID-19. 2,3 Epidemiological modeling results suggest a potentially high value of wearing face masks by the general public to curtail community transmission of COVID-19. Despite an increasing number of healthcare campaigns to urge community-wide face mask wearing, ${ }^{3,4,6-9}$ individual compliance toward face mask wearing remains problematic, ${ }^{4,5}$ and mask wearing could present extra challenges for introducing extra stuffy and sweaty feelings in the tropical climate.

Scholars have noticed different receptiveness to face mask wearing across countries. ${ }^{9}$ For example, in East Asia, face mask wearing can be ubiquitous and was easily made mandatory quickly in the pandemic. ${ }^{6-8,10}$ In many countries, many people are reluctant to and/or oppose wearing face masks because they regard the wearing of face masks as a symbol of individual freedom. ${ }^{6-8}$ However, to date there remains a void on research that assesses which subgroups of the general population are more or less likely to wear face masks. Research on the individual-level heterogeneity in face mask wearing within the same country is especially important because such research can help identify the less-compliant groups under the same policy to enable the deployment of more targeted health information campaigns on mask wearing during the ongoing COVID-19 crisis and future pandemics. Under the prolonged COVID-19 pandemic, we need evidence-based research to generate more targeted actions to get the most out of the thin resources compared with the scale of the pandemic, and hence the identification of the risk factors of mask wearing noncompliance behaviors becomes even more relevant and important.

*Address correspondence to Stephen X. Zhang, University of Adelaide, 9-27 Nexus10 Tower, 10 Pulteney St., Adelaide SA 5000, Australia. E-mail: stephen.x.zhang@gmail.com

†These authors are co-first authors of this article.
To date, there have been only four publications on the individual heterogeneity to wear masks, and all these papers are distinct from our study in their foci. ${ }^{11-14}$ Among the four publications, Hao et al. ${ }^{11}$ used state-level variables such as the death rate across 10 states in the United States to predict individuals' mask wearing. Kim et al. ${ }^{12}$ and Barcelo and Sheen ${ }^{13}$ studied the antecedents of mask wearing by the social norms in Korea and Spain, respectively. ${ }^{12,13}$ Cherry et al. ${ }^{14}$ ran an online experiment with participants from an M-Turk panel to identify public health messaging and personal experience on the acceptance of mask wearing. None of them had the same focus as our study. Furthermore, the four studies were in the USA, Korea, and Spain, and our setting of Malaysia represents the first country in the tropics with a much warmer climate, which may be relevant to mask wearing as well.

To that end, this study investigates individual adults' levels of face mask wearing behavior during the COVID-19 pandemic and explores several predictors based on demographic factors and hygienic factors.

We conducted a survey in May 2020, before Malaysia implemented mandatory mask-wearing in August 2020. The Malaysian Ministry of Health has been advocating wearing face masks as a complementary personal protective device against COVID-19 and has conducted many broad health campaigns with face mask wearing as an essential element. ${ }^{15}$ However, face mask wearing was not mandatory at the time of the study.

Following previous studies, ${ }^{11-14}$ we conducted a crosssectional design because almost all the research on the risk factors is cross-sectional during the COVID-19 crisis $^{16}$ because the COVID-19 crisis makes it very challenging to pursue other designs. The questionnaire is available in all three major languages (i.e., Malay, Mandarin, and English) in Malaysia. The cross-sectional survey was implemented online, which was a safe and feasible way of collecting data during the pandemic, by a two-stage stratified sampling in terms of geographical area, ethnicity, sex, and age, in line with similar studies. To minimize biases, we followed the standard survey approaches (i.e., no social pressure to influence responses, no questions that provoke defensiveness or threaten esteem, no payoff or cost for particular responses). To avoid common method bias, multi-item questions were used to ensure no priming, and there was no overlapping among questions for different constructs. Participation in this survey was voluntary, and 
TABLE 1

Descriptive statistics of respondents

\begin{tabular}{|c|c|c|}
\hline Variables & Frequency (\%) & Mean (SD) \\
\hline \multicolumn{3}{|l|}{ Sex } \\
\hline Male & 345 (48.7) & \\
\hline Female & 363 (51.3) & \\
\hline \multicolumn{3}{|l|}{ Age bracket, years } \\
\hline $19-29$ & 101 (14.3) & \\
\hline 30-39 & $213(30.1)$ & \\
\hline $40-49$ & 200 (28.2) & \\
\hline $50-59$ & 159 (22.5) & \\
\hline$\geq 60$ & $35(4.9)$ & \\
\hline \multicolumn{3}{|l|}{ Number of children in the household } \\
\hline 0 & $335(47.3)$ & \\
\hline 1 & 119 (16.8) & \\
\hline 2 & 112 (15.8) & \\
\hline 3 & 83 (11.7) & \\
\hline 4 & $41(5.8)$ & \\
\hline 5 & $14(2.05)$ & \\
\hline$\geq$ & $4(0.6)$ & \\
\hline \multicolumn{3}{|l|}{ Handwashing } \\
\hline Never & $3(0.4)$ & \\
\hline Rarely ( $<10 \%$ of the time) & $17(2.4)$ & \\
\hline Occasionally ( $\sim 30 \%$ of the time) & $60(8.5)$ & \\
\hline Sometimes ( $\sim 50 \%$ of the time) & $106(15.0)$ & $5.3(1.4)$ \\
\hline Frequently ( $\sim 70 \%$ of the time) & $199(28.1)$ & \\
\hline Usually ( $90 \%$ of the time) & 119 (16.8) & \\
\hline Every time & $204(28.8)$ & \\
\hline \multicolumn{3}{|l|}{ Availability of PPE } \\
\hline Never & $7(1.0)$ & \\
\hline Rarely & $11(1.6)$ & \\
\hline Sometimes & $57(8.1)$ & $4.5(0.8)$ \\
\hline Often & $170(24.0)$ & \\
\hline Always & $463(65.4)$ & \\
\hline \multicolumn{3}{|l|}{ Face mask wearing } \\
\hline Never & $4(0.6)$ & \\
\hline Rarely ( $<10 \%$ of the time) & $8(1.1)$ & \\
\hline Occasionally ( $\sim 30 \%$ of the time) & $15(2.1)$ & \\
\hline Sometimes ( $50 \%$ of the time) & $27(3.8)$ & $6.3(1.2)$ \\
\hline Frequently ( $70 \%$ of the time) & $83(11.7)$ & \\
\hline Usually ( $90 \%$ of the time) & 104 (14.7) & \\
\hline Every time & 467 (66.0) & \\
\hline
\end{tabular}

respondents could opt out at any time. Moreover, respondents were assured anonymity and confidentiality of their responses. All respondents consented to the survey, which was ethically approved (\#20200322).

The list of variables collected is shown below, and the detailed scales are in the supplemental material.

Sex: 1 for male and 2 for female

Age: ranged from 21 years old to 71 years old

The number of children in the household: from 1 (none) to 7 (six or more children)

Handwashing: Handwashing was measured from 1 (never) to 7 (every time)
Availability of personal protective equipment (PPE): Given the availability of PPE (personal protective equipment) was an issue early on in the pandemic, the respondents reported the extent to which they had sufficient personal protective equipment PPE from 1 (never) to 5 (always)

Face mask wearing: The respondents reported how frequently they wore facemasks when outside of their residence from 1 (never) to 7 (every time)

The survey yielded 708 valid responses. We used SPSS (v.26) to run a multivariate least-square regression at the significance level of 0.05 .

Table 1 shows the descriptive statistics of the sample (the distribution, mean, and standard deviation of the Likert scales). Overall, this study found a higher level of face mask compliance, even though the data for this study were collected before the mandatory face mask wearing in public places. About two-thirds (66.0\%) of the adults wore masks all the time when outing, and the rest $33.4 \%$ wore them at varying frequencies. A tiny percentage $(0.5 \%)$ never wore masks under the pandemic.

Table 2 shows the regression result to predict individual adults' face mask wearing. Among the demographic factors, sex positively predicted face mask-wearing, but not age and the number of children in the household did not. In terms of personal hygiene factors, both handwashing and the availability of PPE positively predicted face mask wearing. Furthermore, as a robustness test, we added additional controls, such as the education level, ethnicity, income, etc., and the findings remain robust.

Our finding on sex is consistent with recent studies on the sex difference in terms of face mask wearing. A US-based study found that female shoppers were 1.5 times more likely to wear face masks than male shoppers. ${ }^{17}$ A study in Saudi Arabia revealed the same sex difference. ${ }^{18}$ Our results reveal that two key individual-level hygienic factors during epidemics-handwashing and PPE availability-predicted face mask wearing. Our results demonstrate that those who were less likely to practice handwashing or had less PPE available were also less unlikely to practice face mask wearing. The identification of these predictors enables healthcare organizations to identify statistically the less-compliant groups to enable the deployment of more targeted health information campaigns on mask wearing during the ongoing COVID-19 crisis and future pandemics. Such identification of the less-compliant groups enables more targeted actions to get the most out of the thin resources compared with the scale of the pandemic, and hence the identification of the risk factors of mask wearing noncompliance behaviors is crucial in the prolonged pandemic.

This research is not without limitations. To begin with, our survey captured the individual heterogeneity at a single point

TABLE 2

Predictors of face mask-wearing among 708 individual adults

\begin{tabular}{|c|c|c|c|c|c|}
\hline \multirow[b]{2}{*}{ Variables } & \multirow[b]{2}{*}{ Standardized coefficient } & \multirow[b]{2}{*}{$t$} & \multirow[b]{2}{*}{$P$ value } & \multicolumn{2}{|c|}{ 95\% Confidence interval } \\
\hline & & & & Lower bound & Upper bound \\
\hline Sex (female) & 0.078 & 2.339 & 0.020 & 0.029 & 0.333 \\
\hline Age bracket & 0.017 & 0.499 & 0.618 & -0.052 & 0.088 \\
\hline Number of children in household & -0.042 & -1.261 & 0.208 & -0.088 & 0.019 \\
\hline Handwashing frequency & 0.156 & 4.355 & 0.000 & 0.070 & 0.186 \\
\hline PPE availability & 0.395 & 11.273 & 0.000 & 0.477 & 0.678 \\
\hline
\end{tabular}

PPE $=$ personal protective equipment. 
in the middle of the pandemic. It would be ideal to learn how individuals' mask wearing behaviors change as the pandemic continues to develop. In terms of methodology, the Webbased design means that people with no internet access and limited computer literacy were not surveyed, which made it hard for us to include the older population. Our study used a cross-sectional survey, which is popular in identification studies in COVID-19, and future studies may use matched or control case datasets. Moreover, other predictors, such as the need to go out, the locations to which individuals venture out, as well as psychological factors (including, but not limited to their individual perception, ${ }^{19}$ perceived risk, ${ }^{20}$ knowledge, perceived benefit, trust, social media, etc.) can be studied as additional predictors of mask wearing. Given the measure of the dependent variable, we used linear regression instead of logistic regression. We call future research to study mask wearing not only by its frequency with linear regression but also by yes or no with logistic regression.

In conclusion, by reporting the heterogeneity of individual adults' face mask wearing and identifying several associated predictors, this study advanced research on mask wearing. The results revealed substantial individual heterogeneity in wearing masks and found that individual's sex, hand washing, and the availability of PPE predicted their face mask wearing. This evidence-based research can help public health organizations to identify the less-compliant groups to enable the deployment of more targeted health information campaigns on mask wearing during the ongoing COVID-19 crisis and future pandemics.

Received May 26, 2021. Accepted for publication June 30, 2021.

Published online August 9, 2021.

Note: Supplemental appendix appears at www.ajtmh.org.

Acknowledgments: The study was conducted according to the guidelines of the Declaration of Helsinki, and approved by the Institutional Review Board (or Ethics Committee) of Tsinghua University (20200322). The American Society of Tropical Medicine and Hygiene has waived the Open Access fee for this article due to the ongoing COVID-19 pandemic.

Financial support: This work was supported by the National Natural Science Foundation of China (grant 71772103).

Authors' addresses: Stephen X. Zhang, University of Adelaide, Adelaide, South Australia, Australia, E-mail: stephen.x.zhang@gmail. com. Kim Hoe Looi, Xiamen University Malaysia, Sunsuria city, Sepang, Selangor, Malaysia, E-mail: gideon_looi@hotmail.com. Nicolas Li, University of Dundee, Dundee, Scotland, UK, E-mail: NLi001@dundee.ac.uk. Xue Wan, Tongji University, Shanghai, China, E-mail: wanxue@tongji.edu.cn. Jizhen Li, Tsinghua University, Beijing, China, E-mail: lijzh@sem.tsinghua.edu.cn

This is an open-access article distributed under the terms of the Creative Commons Attribution (CC-BY) License, which permits unrestricted use, distribution, and reproduction in any medium, provided the original author and source are credited.

\section{REFERENCES}

1. CDC, 2019. Cover Your Mouth and Nose with a Mask when around Others. Available at: https://www.cdc.gov/ coronavirus/2019-ncov/prevent-getting-sick/prevention.html. Accessed November 24, 2020.
2. World Health Organization, 2020. Rational Use of Personal Protective Equipment for Coronavirus Disease (COVID-19) and Considerations during Severe Shortages: Interim Guidance. Available at: https://www.who.int/publications/i/ item/rational-use-of-personal-protective-equipment-forcoronavirus-disease-(covid-19)-and-considerations-duringsevere-shortages. Accessed November 11, 2020.

3. World Health Organization, 2020. Advice on the Use of Masks in the Context of COVID-19: Interim Guidance, 5 June 2020. Available at: https://apps.who.int/iris/handle/10665/332293. Accessed November 11, 2020.

4. Cheng VC et al., 2020. The role of community-wide wearing of face mask for control of coronavirus disease 2019 (COVID-19) epidemic due to SARS-CoV-. J Infect 81: 107-114.

5. Eikenberry SE, Mancuso M, Iboi E, Phan T, Eikenberry K, Kuang Y, Kostelich E, Gumel AB, 2020. To mask or not to mask: modeling the potential for face mask use by the general public to curtail the COVID-19 pandemic. Infect Dis Model 5: 293-308.

6. Babak J, Weekes MP, Matheson NJ, 2020. COVID-19: should the public wear face masks? BMJ 369: 1-2.

7. Chen X, Ran L, Liu Q, Hu Q, Du X, Tan X, 2020. Hand hygiene, mask-wearing behaviors, and its associated factors during the COVID-19 epidemic: a cross-sectional study among primary school students in Wuhan, China. Int J Environ Res Public Health 17: 2893.

8. Ma Q, Shan H, Zhang H, Li G, Yang R, Chen J, 2020. Potential utilities of mask-wearing and instant hand hygiene for fighting SARS-CoV-2. J Med Virol 92: 1567-1571.

9. Van Bavel JJ, Baicker K, Boggio PS, Capraro V, Cichocka A, Cikara M, Willer R, 2020. Using social and behavioural science to support COVID-19 pandemic response. Nat Hum Behav 4: 460-471.

10. Greenhalgh T, Schmid MB, Czypionka T, Bassler D, Gruer L, 2020. Face masks for the public during the COVID-19 crisis. BMJ 369: m1435.

11. Hao F, Shao W, Huang W, 2021. Understanding the influence of contextual factors and individual social capital on American public mask wearing in response to COVID-19. Health Place 68: 102537.

12. Kim YJ, Cho J, Kang SW, 2020. Study on the relationship between leisure activity participation and wearing a mask among Koreans during COVID-19 crisis: using TPB model. Int J Environ Res Public Health 17: 7674.

13. Barceló J, Sheen GCH, 2020. Voluntary adoption of social welfare-enhancing behavior: mask-wearing in Spain during the COVID-19 outbreak. PLoS One 15: e0242764.

14. Cherry TL, James AG, Murphy J, 2021. The impact of public health messaging and personal experience on the acceptance of mask wearing during the COVID-19 pandemic. J Econ Behav Organ 187: 415-430.

15. Mohd H, 2020. As COVID-19 Spikes, Health DG Gives 'Sermon' Stressing SOP Compliance. Available at: https://www. malaysiakini.com/news/545097. Accessed October 3, 2020.

16. Hearne BN, Niño MD, 2021. Understanding how race, ethnicity, and gender shape mask-wearing adherence during the COVID-19 pandemic: evidence from the COVID impact survey. $J$ Racial Ethn Health Disparities, 1-8 (E-pub ahead of print).

17. Long WJ, 2011. Pandemics and Peace: Public Health Cooperation in Zones of Conflict. Washington, DC: US Institute of Peace Press.

18. Haischer MH et al., 2020. Who is wearing a mask? Gender-, age-, and location-related differences during the COVID-19 pandemic. PLoS One 15: e0240785.

19. Dai H, Zhang SX, Looi KH, Su R, Li J, 2020. Perception of health conditions and test availability as predictors of adults' mental health during the COVID-19 pandemic: a survey study of adults in Malaysia. Int J Environ Res Public Health 17: 5498.

20. Yan J, Kim S, Zhang SX, Foo MD, Alvarez-Risco A, Del-AguilaArcentales S, Yáñez JA, 2021. Hospitality workers' COVID-19 risk perception and depression: a contingent model based on transactional theory of stress model. Int J Hospit Manag 95: 102935. 